\title{
In this issue
}

\section{Diet quality indices and the need for more validation studies}

Fundamental to the study and practice of nutrition is the understanding that nutritional status and nutrition-related health are not just the result of exposure to individual nutrients found in the diet, but also the complexity of interactions between nutrients and non-nutrient components in foods. This has prompted the relatively recent study of diet quality as an index for assessing the relationship between dietary exposures and health outcomes. Diet quality measurement involves assessing how well diet patterns align with national dietary guidelines and how diverse the variety of healthy choices is within core food groupings. In this issue, Wirt and Collins ${ }^{(1)}$ present a comprehensive review of diet quality indices and find a range of limitations when comparing and interpreting results, supporting their recommendation that more research on validating diet quality indices is needed.

\section{The first 2 years critical}

Nutrition in the first 2 years of life has a long-lasting effect on individual development and health, making dietary assessment during this life-stage an important practice and research focus. Castro et al. ${ }^{(2)}$ present a cross-sectional study of dietary practices and nutritional status of a sample of 0-2-year-old children from Brazilian Amazonia. They provide further evidence to support exclusive breast-feeding in the first 6 months of life and promote the greater emphasis on enhancing the bioavailability of iron in complementary feeds.

\section{Infant feeding and minimisation of HIV transmission}

The transmission of HIV through breast milk has presented significant challenges for the promotion of infant nutrition and the minimisation of mother-to-child HIV transmission. In this issue, Chopra et al. ${ }^{(3)}$ describe a rapid assessment of infant feeding support to HIV-positive women accessing prevention of mother-to-child transmission services in Africa. They identify limited capacity for infant feeding supports and a widespread misunderstanding of the HIV transmission risks with breast-feeding.

\section{The power of educating women}

Many studies of the determinants of breast-feeding initiation have observed the important effects of social class and maternal education. A paper by Skafida ${ }^{(4)}$ in this issue, describing a study of the relative importance of social class and maternal education on breast-feeding initiation in Scotland, suggests that maternal education is a more informative, accurate and useful lens through which to understand and explain breast-feeding take-up.

Roger Hughes Deputy Editor

\section{References}

1. Wirt A \& Collins CE (2009) Diet quality - what is it and does it matter? Public Health Nutr 12, 2473-2492.

2. Castro TG, Baraldi LG, Muniz PT et al. (2009) Dietary practices and nutritional status of 0-24-month-old children from Brazilian Amazonia. Public Health Nutr 12, $2335-2342$.

3. Chopra M, Doherty T, Mehatru S et al. (2009) Rapid assessment of infant feeding support to HIV-positive women accessing prevention of mother-to-child transmission services in Kenya, Malawi and Zambia. Public Health Nutr 12, 2323-2328.

4. Skafida V (2009) The relative importance of social class and maternal education for breast-feeding initiation. Public Health Nutr 12, 2285-2292. 